\title{
Pronóstico en pacientes con síndrome coronario agudo
}

Risk prediction in patients suffering acute coronary syndrome

\section{Objetivo}

Estimar el riesgo de muerte y/o infarto agudo de miocardio (IAM) en pacientes con síndrome coronario agudo (SCA).

\section{Diseño}

Cohorte prospectiva.

\section{Lugar}

94 hospitales de Europa, Norteamerica, Sudamerica y Oceanía.

\section{Población}

Para desarrollar la regla de predicción clínica* (RPC) fueron seleccionados 21688 pacientes con SCA que habían sido enrolados en el registro GRACE entre 1999 y 2005 y asignados a alguna de las siguientes categorías: IAM con supradesnivel del segmento ST ( $\uparrow \mathrm{ST}$ ), IAM sin $\uparrow S T$, angina inestable.
Fox K y col. BMJ. 2006 Nov 25; 333(7578):1091. Epub 2006 Oct 10. Evaluación de los factores pronósticos

Variables clínicas y demográficas, hallazgos electrocardiográficos, antecedentes y características clínicas al ingreso.

\section{Medición de resultados principales}

Mortalidad y mortalidad más IAM no fatal durante la internación y a los seis meses del alta. La RPC fue validada prospectivamente* en otros 22122 pacientes del mismo registro y en 12142 del estudio GUSTOIIb .

\section{Resultados}

Falleció el 9,4\% de los pacientes de la cohorte de derivación* $(4,9 \%$ en el hospital) y el $15,8 \%$ se infartó entre la presentación y los seis meses del alta. En el subgrupo de validación* falleció el $9 \%$ (4,3\% en el hospital) y $4,3 \%$ se infartó durante el mismo período. El $90 \%$ de la variabilidad pronóstica a los seis meses puede ser predicha con las variables descriptas en las tablas 1 y 2.

Tabla 1: valor pronóstico de las diferentes variables al ingreso luego del análisis multivariable.

\begin{tabular}{|c|c|c|c|c|c|c|c|c|c|c|c|}
\hline \multirow{2}{*}{\multicolumn{2}{|c|}{$\begin{array}{l}\text { Predictores al ingreso } \\
\text { hospitalario }\end{array}$}} & \multicolumn{2}{|c|}{$\begin{array}{l}\text { Pronóstico a los seis meses del } \\
\text { ingreso en } \mathrm{HR}^{\star} \text { (IC95\%) }\end{array}$} & \multirow{2}{*}{\multicolumn{8}{|c|}{ Puntuación de cada variable al ingreso hospitalario }} \\
\hline & & \multirow{3}{*}{\begin{tabular}{|c|} 
Muerte \\
$2,60(2,0-3,32)$ \\
$100-(1-60,1)$ \\
\end{tabular}} & \multirow{3}{*}{\begin{tabular}{|c|} 
Muerte o IAM no fatal \\
$2,20(1,76-2,63)$ \\
$1,25(1,21-1,29)$ \\
\end{tabular}} & & & & & & & & \\
\hline \multicolumn{2}{|c|}{ Paro cardíaco } & & & \multicolumn{8}{|c|}{39} \\
\hline \multicolumn{2}{|l|}{ Edad } & & & $<30: 0$ & 30-39: 8 & 40-49: 25 & 50-59: 41 & 60-69: 58 & 70-79: 75 & 80-99: 91 & $>99: 100$ \\
\hline \multicolumn{2}{|c|}{ Serología de injuria miocard. } & $1,60(1,42-1,78)$ & $1,70(1,60-1,87)$ & \multicolumn{8}{|c|}{14} \\
\hline \multicolumn{2}{|c|}{ Desviación segmento ST } & $1,60(1,41-1,88)$ & & \multicolumn{8}{|c|}{28} \\
\hline \multicolumn{2}{|c|}{ Clase de Killip y Kimball ${ }^{2}$} & $1,50(1,41-1,62)$ & $1,40(1,30-1,46)$ & \multicolumn{2}{|r|}{ I: 0} & \multicolumn{2}{|c|}{ II: 20} & \multicolumn{2}{|c|}{ III: 39} & \multicolumn{2}{|c|}{ IV: 59} \\
\hline \multicolumn{2}{|l|}{ Pulso } & $1,20(1,16-1,31)$ & & $<50: 0$ & 50-69: 3 & 70-89: 9 & 90-109: 15 & 110-149: 24 & 150-199: 38 & \multicolumn{2}{|c|}{$>200: 46$} \\
\hline \multicolumn{2}{|c|}{ Tensión arterial sistólica } & $1,20(1,22-1,30)$ & $1,10(1,07-1,13)$ & $<80: 58$ & 80-99: 53 & 100-119: 43 & 120-139: 34 & 140-159: 24 & 160-199: 10 & \multicolumn{2}{|c|}{$>200: 0$} \\
\hline \multicolumn{2}{|c|}{ Creatininemia al ingreso } & $1,20(1,19-1,29)$ & $1,10(1,08-1,16)$ & 0-0,39: 1 & $0,4-0,79: 4$ & $0,8-1,19: 7$ & 1,2-1,59: 10 & 1,6-1,99: 13 & 2-3,99: 21 & \multicolumn{2}{|c|}{$>4: 28$} \\
\hline \multirow[t]{2}{*}{ Historia de } & Insuf. cardíaca & $1,50(1,32-1,73)$ & $1,30(1,17-1,45)$ & \multirow{2}{*}{\multicolumn{8}{|c|}{ No puntuan para la regla de predicción clínica }} \\
\hline & enf. vasc. perif. & $1,40(1,21-1,62)$ & $1,20(1,08-1,36)$ & & & & & & & & \\
\hline
\end{tabular}

Tabla 2: mortalidad durante la internación según la suma de puntos al ingreso.

\begin{tabular}{c|c|c|c}
\hline Puntaje & Mortalidad & Puntaje & Mortalidad \\
\hline$<60$ & $0,2 \%$ & 160 & $5,4 \%$ \\
\hline 70 & $0,3 \%$ & 170 & $7,3 \%$ \\
\hline 80 & $0,4 \%$ & 180 & $9,8 \%$ \\
\hline 90 & $0,6 \%$ & 190 & $13 \%$ \\
\hline 100 & $0,8 \%$ & 200 & $18 \%$ \\
\hline 110 & $1,1 \%$ & 210 & $23 \%$ \\
\hline 120 & $1,6 \%$ & 220 & $29 \%$ \\
\hline 130 & $2,1 \%$ & 230 & $36 \%$ \\
\hline 140 & $2,9 \%$ & 240 & $44 \%$ \\
\hline 150 & $3,9 \%$ & 250 & $>52 \%$ \\
\hline
\end{tabular}

La RPC incluyó las variables asociadas en forma significativa e independiente al resultado primario. En la cohorte de derivación, el área bajo la curva* fue de 0,82 para la predicción de la mortalidad y 0,70 para la de muerte más infarto, mientras que en la de validación fue de 0,82 y de 0,76 respectivamente. En la validación externa con los pacientes del ensayo Gusto Ilb, fue 0,82 para el grupo muerte.

\section{Conclusiones}

Esta RPC permite categorizar pacientes con SCA para la toma de decisiones.

Palabras clave: pronóstico, síndrome coronario agudo, infarto agudo de miocardio, mortalidad. Key words: prognosis, acute coronary síndrome, acute myocardial infarction, mortality. Fuente de financiamiento: no referida. 


\section{Comentario}

Existen muchas RPC para pacientes con SCA (TIMl ${ }^{3}$, PURSUIT ${ }^{4}$, GUSTO') pero la mayoría proviene de ensayos clínicos controlados y aleatorizados, lo que las hace menos aplicables a la práctica cotidiana. Sin embargo, la que hoy describimos fue desarrollada sobre la base de un registro hospitalario y utiliza variables disponibles en la mayoría de las instituciones de la región. Puede también implementarse cargándolas en una página de Internet (www.outcomes.org/grace) o instalando un programa en una computadora. Una de sus ventajas es que la definición de la variable "evidencia serológica de injuria miocárdica" se aplica a los dife- rentes modos de documentarla (curva de creatin fosfoquinasa o $\mathrm{CPK}, \mathrm{CPK} \mathrm{MB}$, niveles de troponina sérica) lo que hace a esta regla más generalizable en nuestro medio.

\section{Conclusión del comentador}

Esta RPC permitiría estimar con buena precisión la mortalidad de los pacientes internados SCA en nuestros lugares de trabajo.

Figura 1: página de Internet (www.outcomes.org/grace) donde pueden cargarse las variables de los pacientes para estimar su pronóstico.

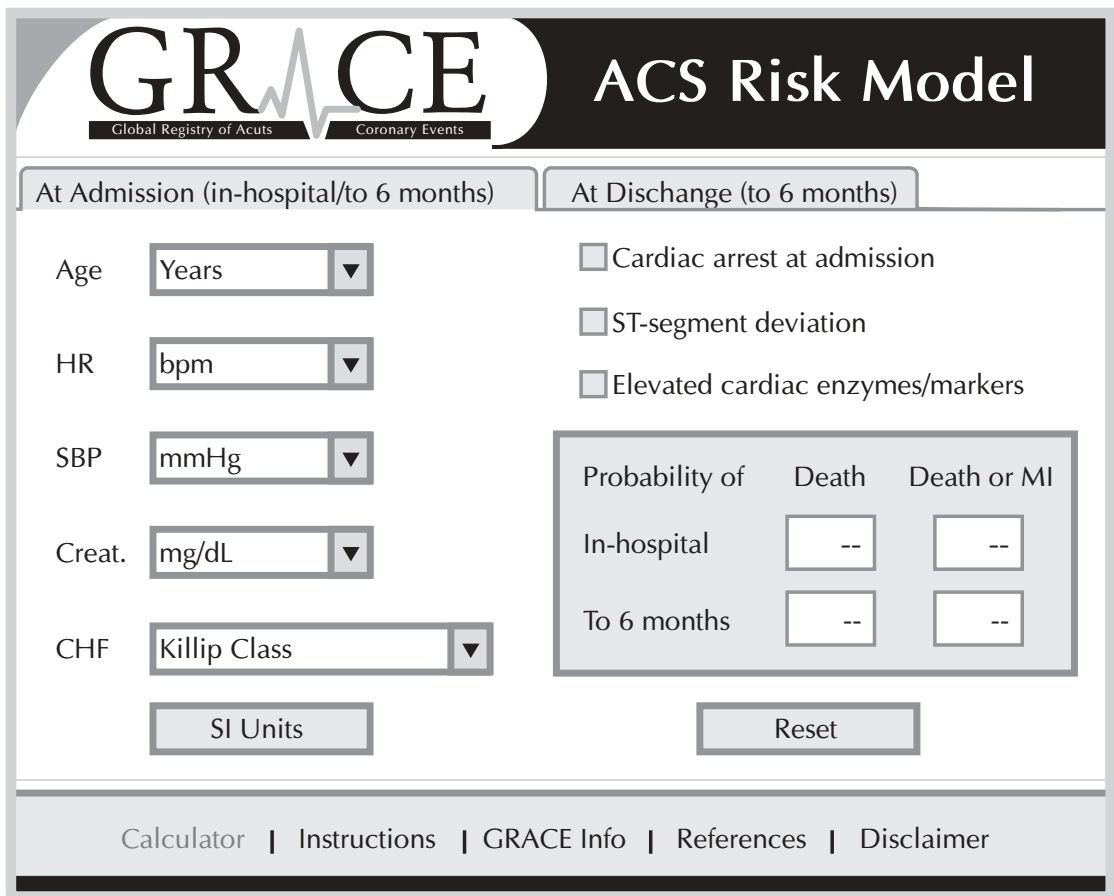

Ver glosario*

Juan Pablo Corso [ Instituto Cardiovascular Buenos Aires. ]

Recibido el 12/01/07 y aceptado el 20/01/2007.

Juan Corso. Pronóstico en pacientes con síndrome coronario agudo. Evid. actual. práct. ambul; 10(1):12-13, ene-feb.2007. Comentado de: Fox.K; Dabbous.O; Goldberg.K. y col. Prediction of risk of death and myocardial infarction in the six months alter presentation with acute coronary síndrome: prospective multinacional obsevational study (GRACE). BMJ.2006. November 25. Vol 333. 1091-4. PMID: 17032691.

Disponible en URL: http://www.bmj.com/cgi/content/full/333/7578/1091 Último acceso: 21/01/07.

Referencias

1. The GUSTO-Ilb Investigators. Acute coronary syndromes in the GUSTO-Ilb trial: prognostic insights and impact of recurrent ischemia.. Circulation 1998 Nov 3;98(18):1860-8.

2. Killip T, 3rd, Kimball, JT. Treatment of myocardial infarction in a coronary care unit. A two year experience with 250 patients. Am J Cardiol 1967; $20: 457$. 3. TIMI risk score for ST-elevation myocardial infarction: A convenient, bedside, clinical score for risk assessment at presentation: An intravenous nPA for treatment of infarcting myocardium early II trial substudy. Circulation 2000 Oct 24;102(17):2031-7.

4. The PURSUIT Trial Investigators. Inhibition of platelet glycoprotein IIb/IIla with eptifibatide in patients with acute coronary syndromes. Platelet Glycoprotein Ilb/Illa in Unstable Angina: Receptor Suppression Using Integrilin Therapy. N Engl J Med 1998 Aug 13;339(7):436-43. 